Algebraic $\&$ Geometric $\mathcal{T}_{\text {opology }}$

Volume 4 (2004) 1125-1144

Published: 25 November 2004

ATG

\title{
Transverse contact structures on Seifert 3-manifolds
}

\author{
PAOLO LisCA \\ Gordana Matić
}

\begin{abstract}
We characterize the oriented Seifert-fibered three-manifolds which admit positive, transverse contact structures.
\end{abstract}

AMS Classification 57R17

Keywords Transverse contact structures, Seifert three-manifolds

\section{Introduction and statement of results}

Foliations and contact structures are arguably at opposite ends of the spectrum of the possible 2-plane fields $\xi$ on a 3 -manifold. While foliations are integrable plane fields, contact structures are totally non-integrable. If the planes in the distribution $\xi$ are given as kernels of a one form $\alpha$, then they form a foliation if $\alpha \wedge d \alpha=0$, while they form a contact structure if everywhere pointwise $\alpha \wedge d \alpha \neq 0$. Eliashberg and Thurston [4] introduced the notion of confoliation, which generilizes the notion of foliation as well as the notion of contact structure. They also showed that a taut foliation can be $C^{0}$-approximated by a fillable contact structure.

The problem of existence of foliations transverse to the $S^{1}$-action on a Seifert fibered 3-manifold $M$ was studied, and almost completely solved, in the 1980's by Eisenbud, Hirsch, Jankins and Neumann who translated, via holonomy, the original problem into a problem about homeomorphisms of the circle 1, 9, 10. The problem was settled by Naimi [14].

The existence of transverse contact structures is clearly a related question. A foliation transverse to the $S^{1}$-action on a Seifert 3 -manifold is taut since any orbit provides a closed loop transverse to the leaves of the foliation. If a transverse foliation $\mathcal{F}$ exists and the underlying manifold is not $S^{1} \times S^{2}$ then, according to Eliashberg and Thurston $4, \mathcal{F}$ can be approximated by a fillable contact structure $\xi$. The approximation is still transverse to the circle action 
on $M$. However, often $M$ supports a transverse tight contact structure even when there are no transverse taut foliations.

Let $p: Y \rightarrow \Sigma_{g}$ be an oriented three-dimensional circle bundle with Euler class $e(Y)$ and base of genus $g$. Here $g \in \mathbb{Z}$ is defined so that

$$
\chi\left(\Sigma_{g}\right)=\left\{\begin{array}{l}
2-2 g \text { if } \Sigma_{g} \text { is orientable } \\
2+g \text { if } \Sigma_{g} \text { is non-orientable }
\end{array}\right.
$$

where $\chi\left(\Sigma_{g}\right)$ is the Euler characteristic of $\Sigma_{g}$. Note that, with this convention, $\Sigma_{g}$ is orientable for $g \geq 0$ and non-orientable for $g<0$. Milnor and Wood studied the case of foliations transverse to the fibers of $p$. According to [12, 20, $Y$ carries a transverse foliation if and only if one of the following holds:

- $\chi\left(\Sigma_{g}\right) \leq 0$ and $|e(Y)| \leq-\chi\left(\Sigma_{g}\right)$,

- $\chi\left(\Sigma_{g}\right) \geq 0$ and $e(Y)=0$.

More recently, Giroux [6] and Sato and Tsuboi [19] proved that $Y$ admits a positive, transverse contact structure if and only if one of the following holds:

- $\chi\left(\Sigma_{g}\right) \leq 0$ and $e(Y) \leq-\chi\left(\Sigma_{g}\right)$,

- $\chi\left(\Sigma_{g}\right)>0$ and $e(Y)<0$.

Let $p: M \rightarrow \Sigma_{g}$ be an oriented three-dimensional Seifert fibration with base of genus $g$ and normalized (in the sense of [16]) Seifert invariants

$$
\left\{b, g ;\left(\alpha_{1}, \beta_{1}\right), \ldots,\left(\alpha_{r}, \beta_{r}\right)\right\} \text {. }
$$

Define

$$
e(M):=-b-\sum_{i=1}^{r} \frac{\beta_{i}}{\alpha_{i}}, \quad e_{0}(M):=-b-r, \quad \text { and } \quad \Gamma(M):=\left(\gamma_{1}, \ldots, \gamma_{r}\right),
$$

where

$$
\gamma_{i}:=1-\frac{\beta_{i}}{\alpha_{i}}, \quad i=1, \ldots, r
$$

Recall that the normalized Seifert invariants of $-M$ are

$$
\left\{-b-r, g ;\left(\alpha_{1}, \alpha_{1}-\beta_{1}\right), \ldots,\left(\alpha_{r}, \alpha_{r}-\beta_{r}\right)\right\} \text {. }
$$

Therefore,

$e(-M)=-e(M), \quad e_{0}(-M)=-e_{0}(M)-r=b, \quad \Gamma(-M)=\left(1-\gamma_{1}, \ldots, 1-\gamma_{r}\right)$.

Definition 1.1 We say that $\Gamma=\left(\gamma_{1}, \ldots, \gamma_{r}\right) \in(\mathbb{Q} \cap(0,1))^{r}$ is realizable if $r \geq 3$ and there exist a permutation $\sigma \in \mathcal{S}_{r}$ and coprime integers $m>a>0$ such that:

$$
\gamma_{\sigma(1)}<\frac{a}{m}, \quad \gamma_{\sigma(2)}<\frac{m-a}{m}, \quad \gamma_{\sigma(3)}, \ldots, \gamma_{\sigma(r)}<\frac{1}{m}
$$


The main results of Eisenbud, Hirsch, Jankins, Neumann and Naimi on the existence of transverse foliations can be summarized in the following statement.

Theorem 1.2 1, 10, 14] Let $p: M \rightarrow \Sigma_{g}$ be an oriented three-dimensional Seifert fibration as above. Then, $M$ carries a smooth foliation transverse to the fibration if and only if one of the following holds:

(a) $e_{0}(M) \leq-\chi\left(\Sigma_{g}\right)$ and $e_{0}(-M) \leq-\chi\left(\Sigma_{g}\right)$

(b) $g=0$ and $e(M)=e(-M)=0$

(c) $g=0, e_{0}(M)=-1$ and $\Gamma(M)$ is realizable

(d) $g=0, e_{0}(-M)=-1$ and $\Gamma(-M)$ is realizable

Warning The reader should be aware of a difference in conventions: the quantity denoted by ' $b$ ' in [10] corresponds to $-b$ in our present notation.

The main result of this paper is the following theorem, giving necessary and sufficient conditions for the existence of contact structures transverse to the fibers of a Seifert fibration:

Theorem 1.3 Let $p: M \rightarrow \Sigma_{g}$ be an oriented three-dimensional Seifert fibration as above. Then, $M$ carries a positive contact structure transverse to the fibers of $p$ if and only if one of the following holds:

(a) $e_{0}(M) \leq-\chi\left(\Sigma_{g}\right)$

(b) $g=0, r \leq 2$ and $e(M)<0$

(c) $g=0, e_{0}(M)=-1$ and $\Gamma(M)$ is realizable.

In view of the Eliashberg-Thurston's approximation theorem, one might wonder why Condition (b) from Theorem 1.2 does not appear explicitely in the statement of Theorem [1.3. The reason is that $e(M)=e(-M)=0$ implies $e_{0}(M) \leq-1$ and $e_{0}(-M) \leq-1$. If both inequalities are strict, case (a) of Theorem 1.3 holds for both $M$ and $-M$. If both inequalities are equalities, then $r=2$ and it is easy to check that $M=S^{1} \times S^{2}$, therefore the Eliashberg-Thurston's perturbation theorem cannot be applied. If $e_{0}(M)=-1$ and $e_{0}(-M) \leq-2$, (a) of Theorem 1.3 holds for $-M$ and, since $r \geq 3$, it follows from [10, 14] that (c) of Theorem 1.3] holds for $M$. Similarly, if $e_{0}(M)=-2$ and $e_{0}(-M) \leq-1$ then (a) of Theorem 1.3 holds for $M$ and (c) of Theorem 1.3 for $-M$.

It should be mentioned that Honda [7] uses monodromy arguments (parallel to the ones used in the foliation case) to study the existence of transverse contact structures, obtaining results similar to ours. 
Our approach is completely different. We show that the conditions of Theorem 1.3 are imposed on a Seifert 3-manifold $M$ by the existence of a transverse contact structure $\xi$ because of a certain property of a specific symplectic filling $X_{M}$ of $(M, \xi)$. The property is simply the fact that surfaces embedded in $X_{M}$ and any of its blowdowns satisfy the adjunction inequalities.

To prove Theorem 1.3 when $\Sigma_{g}$ is orientable, we look at $M$ as the boundary of an equivariant plumbing $X_{M}$ built according to a weighted star $\Gamma$ of Figure 1 as described in 16. McCarthy and Wolfson 13. showed that $X_{M}$ carries a symplectic structure such that the orbits of the $S^{1}$-action on $M$ are tangent to the kernel of the restriction of the symplectic form. For any transverse contact structure $\xi$ on $M$, this makes $X_{M}$ a symplectic filling of $(M, \xi)$. To prove that the conditions of Theorem 1.3 are necessary, we impose that the adjunction inequalities be satisfied by surfaces embedded in $X_{M}$ and, if the central node of $\Gamma$ is a $(-1)$-sphere, into successive blow-downs of $X_{M}$.

It turns out that the most difficult case to analyze is case (c) of Theorem 1.3. involving the "realizability" condition. It is very interesting to see it appear from this new angle. In fact, one may think of the proof of Theorem 1.3 as a way of checking whether a given $\Gamma(M)=\left(\gamma_{1}, \ldots, \gamma_{r}\right)$ is realizable. In short, $\Gamma(M)$ is realizable if and only if in the successive blow-downs of the plumbed manifold $X_{M}$ there are no obvious surfaces violating the adjunction inequality.

The case when $\Sigma_{g}$ is non-orientable is reduced to the orientable case by pulling back the fibration $M \rightarrow \Sigma_{g}$ to the orientable double cover of $\Sigma_{g}$.

To prove the existence of a transverse contact structure under the assumptions of Theorem 1.3, we either use the results on foliations and the EliashbergThurston approximation result, or we construct the contact structure directly.

The paper is organized as follows. In Section 2 we describe in detail the symplectic filling $X_{M}$, and we collect its necessary properties. In Section 3 we characterize those Seifert $3-$ manifolds which admit $S^{1}$-invariant transverse contact structures, and in Section 4 we prove Theorem 1.3

\section{Symplectic fillings}

Let $p: M \rightarrow \Sigma_{g}$ be a Seifert 3-manifold with orientable base and Seifert invariants

$$
\left\{b, g ;\left(\alpha_{1}, \beta_{1}\right), \ldots,\left(\alpha_{r}, \beta_{r}\right)\right\}
$$


Suppose that

$$
\frac{\alpha_{i}}{\alpha_{i}-\beta_{i}}=\left[b_{i, 1}, \ldots, b_{i, s_{i}}\right], \quad i=1, \ldots, r
$$

where

$$
\left[b_{1}, \ldots, b_{k}\right]:=b_{1}-\frac{1}{b_{2}-\frac{1}{\ddots \cdot-\frac{1}{b_{k}}}}, \quad b_{1}, \ldots, b_{k} \geq 2
$$

Note that, since we assume $0<\beta_{i}<\alpha_{i}$, there is a unique continued expansion with all $b_{i, j} \geq 2$.

By [16. Chapter 2], $M$ is isomorphic (as a 3 -manifold with $S^{1}$-action) to the boundary of a 4-manifold with $S^{1}$-action $X_{M}$ obtained by equivariant plumbing according to the graph $\Gamma$ of Figure 1, where the central vertex represents a disk bundle with Euler number $e_{0}(M)$ over a surface of genus $g$, and all the other vertices represent disk bundles over the sphere with Euler numbers $-b_{i, j}$.

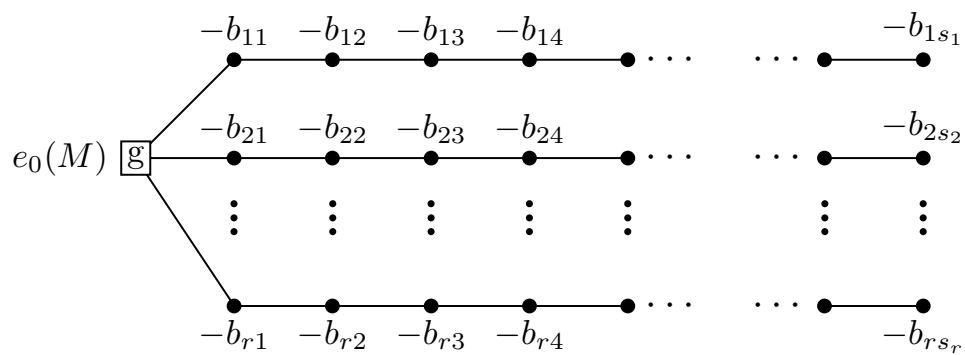

Figure 1: The plumbing $X_{M}$

Theorem 2.1 (13]) The $S^{1}$-manifold $X_{M}$ carries a symplectic form $\omega$ such that every orbit of the $S^{1}$-action on $\partial X_{M}$ is tangent to the kernel of $\left.\omega\right|_{\partial X_{M}}$.

Recall that a symplectic filling of a closed contact 3 -manifold $(M, \xi)$ is a symplectic 4-manifold $(X, \omega)$ such that (i) $X$ is oriented by $\omega \wedge \omega$, (ii) $\partial X=M$ as oriented manifolds, and (iii) $\left.\omega\right|_{\xi} \neq 0$ at every point of $M$.

Corollary 2.2 Let $p: M \rightarrow \Sigma_{g}$ be an oriented Seifert fibered 3-manifold with orientable base $\Sigma_{g}$. Let $\xi$ be a a positive contact structure on $M$ transverse to the fibration. Then, $\left(X_{M}, \omega\right)$ from Theorem 2.1] is a symplectic filling of $(M, \xi)$. Moreover, $\xi$ is universally tight. 
Proof The first part follows immediately from the definitions together with Theorem 2.1. For the second part, recall that the fundamental group of a Seifert 3-manifold is residually finite [8, pp. 176-177]. Therefore, in order to prove that the contact structure is universally tight it is enough to show that the pull-back of the contact structure to every finite cover is tight. This follows from the first part of the statement, because every finite cover is still Seifert fibered, the pulled-back structure is transverse, and fillable contact structures are tight [2].

Corollary 2.3 Let $p: M \rightarrow \Sigma_{g}$ be an oriented Seifert fibered 3-manifold with orientable base $\Sigma_{g}$. Suppose that $M$ carries a positive contact structure transverse to the fibration. Let $S \subset X_{M}$ be an oriented surface of genus $g(S)$ smoothly embedded in the 4-manfild $X_{M}$ given by Figure 1, Then,

$$
\left\{\begin{array}{l}
S \cdot S \leq-1 \quad \text { if } \quad g(S)=0 \\
S \cdot S \leq-\chi(S) \quad \text { if } \quad g(S)>0 .
\end{array}\right.
$$

Proof Let $\xi$ be a a positive contact structure on $M$ transverse to the fibration. By Corollary 2.2 $(M, \xi)$ has a symplectic filling of the form $\left(X_{M}, \omega\right)$. By [3], $\left(X_{M}, \omega\right)$ can be compactified to a closed, symplectic 4-manifold $\widehat{X}_{M}$. Moreover, up to adding a suitable Stein cobordism to $X_{M}$ (using e.g. [5. Theorem 2.5]) it can be arranged that $b_{2}^{+}\left(\widehat{X}_{M}\right)>1$. Since the stated inequalities are satisfied by surfaces in $\widehat{X}_{M}$ [17], the conclusion follows.

\section{$3 \quad S^{1}$-invariant structures}

This section consists of the following proposition, which characterizes the oriented Seifert 3 -manifolds carrying positive, $S^{1}$-invariant transverse contact structures.

Proposition 3.1 Let $p: M \rightarrow \Sigma_{g}$ be an oriented Seifert 3-manifold. Then, $M$ carries a positive, $S^{1}$-invariant transverse contact structure if and only if $e(M)<0$.

Proof Suppose that $M$ has Seifert invariants

$$
\left\{b, g ;\left(\alpha_{1}, \beta_{1}\right), \ldots,\left(\alpha_{r}, \beta_{r}\right)\right\} .
$$

Let $a=\alpha_{1} \cdots \alpha_{r}$. The cyclic group $\mathbb{Z} / a \mathbb{Z}$ acts on $M$ when it is regarded as a subgroup of $S^{1}$. The quotient $M^{\prime}=M / \mathbb{Z} / a \mathbb{Z}$ is a genuine $S^{1}$-bundle over the 
same base $\Sigma_{g}$. Moreover, by [15, Theorem 1.2],

$$
e\left(M^{\prime}\right)=a e(M)
$$

Thus, if $e(M)<0$ then $e\left(M^{\prime}\right)<0$ and by [6, 19] $M^{\prime}$ carries an $S^{1}$-invariant contact structure $\xi$ transverse to the fibers. The pull-back of $\xi$ to $M$ is also $S^{1}$ invariant and tranverse, therefore we have proved the first half of the statement.

Now let $\xi$ be an $S^{1}$-invariant transverse contact structure on $M$. We claim that there exists an induced $S^{1}$-invariant transverse contact structure $\bar{\xi}$ on $M^{\prime}$. By [6, 19] and Equation (3.1), proving the claim clearly suffices to finish the proof.

To prove the claim, we first argue locally around a singular fiber. Recall that a neighborhood of a singular fiber $F$ is of the form

$$
\left(D^{2} \times S^{1}\right) / \mathbb{Z} / p \mathbb{Z}
$$

where the generator $g \in \mathbb{Z} / p \mathbb{Z}$ acts by $(x, t) \mapsto\left(x g, t g^{q}\right)$ for some $0<q<p$ coprime with $p$. The contact structure $\xi$ lifts to a contact structure $\tilde{\xi}$ on $D \times S^{1}$ which is $\mathbb{Z} / p \mathbb{Z}$-invariant and $S^{1}$-invariant under the standard $S^{1}$ action on the second factor. Since $\xi$ is transverse to the singular fiber $F$, after a suitable change of trivialization of the neighborhood of $F \tilde{\xi}$ is given as the zero set of a 1 -form $\alpha+d \theta$, where $d \theta$ is the standard angular 1 -form on $S^{1}$, $\alpha \in \Omega^{1}\left(D^{2}\right)$ is $S^{1}$-invariant and $d \alpha \in \Omega^{2}\left(D^{2}\right)$ is a volume form.

Since the $\mathbb{Z} / p \mathbb{Z}$-action and the $\mathbb{Z} / a \mathbb{Z}$-action commute, the quotient map from $D^{2} \times S^{1}$ onto a neighorhood of the image $F^{\prime} \subset M^{\prime}$ of $F$ can be factored as follows:

$$
D^{2} \times S^{1} \stackrel{f}{\rightarrow}\left(D^{2} \times S^{1}\right) / \mathbb{Z} / a \mathbb{Z} \stackrel{g}{\rightarrow}\left(\left(D^{2} \times S^{1}\right) / \mathbb{Z} / a \mathbb{Z}\right) / \mathbb{Z} / p \mathbb{Z}
$$

Here $\mathbb{Z} / a \mathbb{Z}$ acts freely on the second factor as a subgroup of $S^{1}$, and after the identification $\left(D^{2} \times S^{1}\right) / \mathbb{Z} / a \mathbb{Z} \cong D^{2} \times S^{1}, \mathbb{Z} / p \mathbb{Z}$ acts by rotations on the first factor. Since $\alpha$ and $d \theta$ are $S^{1}$-invariant, $\tilde{\xi}$, and therefore $\xi$, descends to a smooth contact structure $\bar{\xi}$ on a neighborhood of $F^{\prime}$. Since $\xi$ also descends to a transverse $S^{1}$-invariant contact structure when restricted to the complement of the the singular fibers, the claim is proved. 


\section{The proof of Theorem 1.3}

\subsection{Sufficiency of the conditions}

The case $\chi\left(\Sigma_{g}\right) \leq 0$

Suppose that the inequality stated in case (a) of Theorem 1.3 holds. Let us first assume that $e_{0}(-M) \leq-\chi\left(\Sigma_{g}\right)$. Then, it follows from case (a) of Theorem 1.2 that $M$ carries a smooth foliation $\mathcal{F}$ transverse to the Seifert fibration. Moreover, $M \neq S^{1} \times S^{2}$ because $\chi\left(\Sigma_{g}\right) \leq 0$ implies that the fundamental group of $\Sigma_{g}$ is non-trivial, and this is incompatible with $M=S^{1} \times S^{2}$. Therefore, by [4] there exist positive contact structures arbitrarily $C^{0}$-near $\mathcal{F}$, hence transverse to the fibration.

If $e_{0}(-M)>-\chi\left(\Sigma_{g}\right)$, take $N$ to be the Seifert fibered 3-manifold with Seifert invariants

$$
\left(-\chi\left(\Sigma_{g}\right), g ;\left(\alpha_{1}, \beta_{1}\right), \ldots,\left(\alpha_{r}, \beta_{r}\right)\right)
$$

Since

$$
e_{0}(N)=\chi\left(\Sigma_{g}\right)-r \leq-\chi\left(\Sigma_{g}\right)
$$

$N$ carries a positive contact structure transverse to the fibration. Moreover, $M$ can be obtained from $N$ by a smooth surgery along a regular fiber $f \subset N$ with coefficient

$$
\frac{1}{e_{0}(-M)+\chi\left(\Sigma_{g}\right)}
$$

with respect to the framing induced by the fibration. It is not difficult to check(see [6, Lemma 1.3]) that transverse contact structures extend from the complement of a regular neighborhood of $f$ in $N$ to transverse contact structures on $M$.

The case $\chi\left(\Sigma_{g}\right)>0$, i.e. $g \in\{-1,0\}$

If case (a) of Theorem 1.3 holds, then

$$
e(M)=e_{0}(M)+\sum_{i=1}^{r}\left(1-\frac{\beta_{i}}{\alpha_{i}}\right) \leq-\chi\left(\Sigma_{g}\right)+\sum_{i=1}^{r}\left(1-\frac{\beta_{i}}{\alpha_{i}}\right) .
$$

If $e(M)<0$, then $M$ carries a transverse contact structure by Proposition 3.1 If $e(M) \geq 0$, then Equation (4.1) implies $r \geq 2$ if $g=-1$, and $r \geq 3$ if $g=0$, therefore $M \neq S^{1} \times S^{2}$. Also, since $e(-M)=-e(M) \leq 0$,

$$
e_{0}(-M)=e(-M)-\sum_{i=1}^{r} \frac{\beta_{i}}{\alpha_{i}}<0 \text {, }
$$


therefore $e_{0}(-M) \leq-1$. If (i) $g=-1$ or (ii) $g=0$ and $e_{0}(-M) \leq-2$, then by Theorem 1.2(a) together with the Eliashberg and Thurston's theorem we are done. If $g=0$ and $e_{0}(-M)=-1$ then, since $e(-M) \leq 0$, by 9 , Theorem 1] (see also [10, Theorem 3.1]) and 10, Theorem 3], $-M$, and therefore $M$, supports a smooth foliation $\mathcal{F}$ transverse to the fibration and we conclude as before.

If case (b) of Theorem 1.3 holds, then the conclusion follows by Proposition 3.1

If (c) holds, then we get a transverse contact structure via Theorem 1.2(c) and [4.

\subsection{Necessity of the conditions}

The case $\chi\left(\Sigma_{g}\right) \leq 0$

If $g>0$, observe that the smooth 4 -manifold $X_{M}$ of Section 2 contains a smooth surface $\Sigma_{g}$ of genus $g$ and self-intersection $e_{0}(M)$. If $M$ carries a positive, transverse contact structure, then by Corollary 2.3$] e_{0}(M) \leq-\chi\left(\Sigma_{g}\right)$. Therefore case (a) of Theorem 1.3 holds.

If $g<-1$, let $\widehat{\Sigma}_{g} \rightarrow \Sigma_{g}$ be the orientable double cover of $\Sigma_{g}$, and let $\widehat{M} \rightarrow \widehat{\Sigma}_{g}$ be the pull-back of the fibration $M \rightarrow \Sigma_{g}$. According to [15, Theorem 1.2], $e_{0}(\widehat{M})=2 e_{0}(M)$. If $M$ carries a positive, transverse contact structure then so does $\widehat{M}$. Therefore, since $\widehat{\Sigma}_{g}$ has positive genus we have

$$
e_{0}(\widehat{M}) \leq-\chi\left(\widehat{\Sigma}_{g}\right)=-2 \chi\left(\Sigma_{g}\right)
$$

hence case (a) of Theorem 1.3 holds.

The case $\chi\left(\Sigma_{g}\right)>0$

Suppose first that $g=0$. Then, Corollary 2.3 implies $e_{0}(M) \leq-1$. Hence, either case (a) of Theorem 1.3 holds, or $e_{0}(M)=-1$. Let us assume the latter. If $r=0$ then $e(M)=e_{0}(M)=-1$, therefore case (b) of Theorem 1.3 holds. If $r=1$ or $r=2$, we blow down $(-1)$-spheres in $X_{M}$ as far as possible. The adjuction inequalities imply that no non-negative sphere appears, therefore $X_{M}$ is negative definite and, since $e(M)$ is an eigenvalue of $Q_{X}$ [15, Theorem 5.2], $e(M)<0$. Thus, again case (b) of Theorem 1.3 holds.

When $g=0$, we are left to consider the subcase $g=0$ and $r \geq 3$. Before tackling this subcase we deal with the case $g=-1$. 
If $g=-1$, let $\widehat{M} \rightarrow \widehat{\Sigma}_{g}$ be the pull-back of $M \rightarrow \Sigma_{g}$ under the orientable double cover of $\Sigma_{g}$. By [15, Theorem 1.2] we have

$$
e_{0}(\widehat{M})=2 e_{0}(M) \neq-1 \text {. }
$$

Thus, since $\widehat{\Sigma}_{g}=S^{2}$ and $\widehat{M}$ carries a positive, transverse contact structure, we have $e_{0}(\widehat{M}) \leq-2$. Therefore $e_{0}(M) \leq-1$, and case (a) of Theorem 1.3 holds for $M$.

The subcase $g=0, e_{0}(M)=-1, r \geq 3$

This is the last and hardest subcase. Before delving into the proof, we need some preparation.

\section{Algebraic preliminaries}

Let

$$
\rho \in \mathbb{Q}_{>1}:=\{q \in \mathbb{Q} \mid q>1\} .
$$

Then, there is a unique way of writing $\rho$ as a continued fraction

$$
\rho=\left[a_{1}, \ldots, a_{h}\right], \quad a_{i} \geq 2, i=1, \ldots, h .
$$

It is well-known that if $\frac{p}{q}=\left[a_{1}, \ldots, a_{h}\right]$, with $p, q$ coprime, then $\left[a_{h}, \ldots, a_{1}\right]$ is of the form $\frac{p}{q^{\prime}}$ for some $q^{\prime}$ coprime with $p$.

The set of finite sequences of integers has a natural linear order $\preccurlyeq$ given by the following definition.

\section{Definition 4.1 Declare}

$$
\left(a_{1}, \ldots, a_{h}\right) \preccurlyeq\left(b_{1}, \ldots, b_{k}\right)
$$

if and only if there exists an index $1 \leq j \leq \min (h, k)$ such that

- $a_{i}=b_{i}$ for $i=1, \ldots, j-1$, and

- either (i) $a_{j}<b_{j}$ or (ii) $j=k \leq h$ and $a_{k}=b_{k}$.

For example, we have

$$
(2,2,2) \preccurlyeq(2,2,3) \preccurlyeq(2,2)
$$

Lemma 4.2 Let $\rho, \sigma \in \mathbb{Q}_{>1}$, with $\rho=\left[a_{1}, \ldots, a_{h}\right]$ and $\sigma=\left[b_{1}, \ldots, b_{k}\right]$. Then,

$$
\rho \leq \sigma \quad \Longleftrightarrow \quad\left(a_{1}, \ldots, a_{h}\right) \preccurlyeq\left(b_{1}, \ldots, b_{k}\right) .
$$


Proof Suppose that $\left(a_{1}, \ldots, a_{h}\right) \preccurlyeq\left(b_{1}, \ldots, b_{k}\right)$. Observe that the rational expression $\left[a_{1}, \ldots, a_{h}\right]$ makes sense if $a_{1}, \ldots, a_{h} \in \mathbb{Q}_{>1}$. Let $1 \leq j \leq \min (h, k)$ be such that $a_{i}=b_{i}$ for $1 \leq i \leq j-1$ and either (i) $a_{j}<b_{j}$ or (ii) $j=k \leq h$ and $a_{k}=b_{k}$. If (i) holds then

$$
\left[a_{j}, \ldots, a_{h}\right] \leq a_{j} \leq b_{j}-1<\left[b_{j}, \ldots, b_{k}\right],
$$

therefore we have

$$
\begin{aligned}
\rho & =\left[a_{1}, \ldots, a_{j-1},\left[a_{j}, \ldots, a_{h}\right]\right]= \\
& =\left[b_{1}, \ldots, b_{j-1},\left[a_{j}, \ldots, a_{h}\right]\right]<\sigma=\left[b_{1}, \ldots, b_{j-1},\left[b_{j}, \ldots, b_{k}\right]\right] .
\end{aligned}
$$

If (ii) holds then, since $\left[b_{k}, a_{k+1}, \ldots, a_{h}\right] \leq b_{k}$,

$$
\begin{aligned}
\rho & =\left[a_{1}, \ldots, a_{k}, a_{k+1}, \ldots, a_{h}\right]= \\
& =\left[b_{1}, \ldots, b_{k-1},\left[b_{k}, a_{k+1}, \ldots, a_{h}\right]\right] \leq \sigma=\left[b_{1}, \ldots, b_{k}\right] .
\end{aligned}
$$

Conversely, suppose $\rho \leq \sigma$. If $\left(a_{1}, \ldots, a_{h}\right) \npreceq\left(b_{1}, \ldots, b_{k}\right)$ then $\left(a_{1}, \ldots, a_{h}\right) \neq$ $\left(b_{1}, \ldots, b_{k}\right)$, i.e. $\rho \neq \sigma$ and $\left(b_{1}, \ldots, b_{k}\right) \preccurlyeq\left(a_{1}, \ldots, a_{h}\right)$, which implies $\sigma<\rho$. Therefore we must have $\left(a_{1}, \ldots, a_{h}\right) \preccurlyeq\left(b_{1}, \ldots, b_{k}\right)$.

Consider the involution $\mathbb{Q}_{>1} \rightarrow \mathbb{Q}_{>1}$ which maps $\rho \in \mathbb{Q}_{>1}$ to the only solution $\rho^{\prime} \in \mathbb{Q}_{>1}$ of the equation:

$$
\frac{1}{\rho}+\frac{1}{\rho^{\prime}}=1
$$

Observe that $2=2^{\prime}$, and $\rho_{1} \leq \rho_{2}$ if and only if $\rho_{2}^{\prime} \leq \rho_{1}^{\prime}$. Therefore, the involution maps the interval $(1,2)$ bijectively onto $(2, \infty)$ reversing the standard linear order.

Given

$$
\rho=\left[a_{1}, \ldots, a_{h}\right] \in \mathbb{Q}_{>1},
$$

the Riemenschneider's point diagram [18] $D\left(\rho, \rho^{\prime}\right)$ says how to compute the coefficients in the expansion

$$
\rho^{\prime}=\left[b_{1}, \ldots, b_{k}\right]
$$

The diagram $D\left(\rho, \rho^{\prime}\right)$ consists of $h$ rows of dots, with the $i$-th row consisting of $a_{i}-1$ dots and whose first dot lies under the last dot of the $(i-1)$-st row. Then, $b_{j}$ is given by the number of elements in the $j$-th column of $D\left(\rho, \rho^{\prime}\right)$ increased by one. For example, if $\rho=[3,4,3]$ and $\rho^{\prime}=[2,3,2,3,2]$, the diagram is given by Figure 2 , 


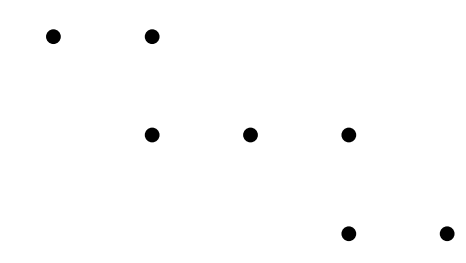

Figure 2: The point diagram $D([3,4,3],[2,3,2,3,2])$

\section{Setting up the stage for the proof}

Recall that we are assuming $g=0, e_{0}(M)=-1$ and $r \geq 3$. We need to show that if $M$ carries a positive, transverse contact structure then $\Gamma(M)=$ $\left(\gamma_{1}, \ldots, \gamma_{r}\right)$ is realizable. Without loss of generality, we may assume

$$
\gamma_{1} \geq \gamma_{2} \geq \cdots \geq \gamma_{r}
$$

Define

$$
\delta_{i}:=\frac{1}{\gamma_{i}}, i=1, \ldots, r .
$$

The realizability of $\Gamma(M)$ is equivalent to the existence of coprime integers $a, m$ such that $1 \leq a<m$ and

$$
\delta_{1}>\frac{m}{a}, \quad \delta_{2}>\frac{m}{m-a}, \quad \delta_{3}, \ldots, \delta_{r}>m .
$$

If $\delta_{1}>2$ then Inequalities (4.2) are satisfied for $m=2, a=1$, and $\Gamma(M)$ is realizable. Therefore we may assume $\delta_{1} \leq 2$. Under this assumption, we can write the continued fraction expansion of $\delta_{1}$ as:

$$
\delta_{1}=\overbrace{2, \ldots, 2}^{n_{1}+1}, n_{2}, \overbrace{2, \ldots, 2}^{n_{3}}, n_{4}, \ldots, n_{2 p}, \overbrace{2, \ldots, 2}^{n_{2 p+1}}]
$$

for some

$$
n_{1}, n_{3}, \ldots, n_{2 p+1} \geq 0, \quad n_{2}, n_{4}, \ldots, n_{2 p} \geq 3 .
$$

Next, we claim that we may also assume $\delta_{2}>2$. In fact, if $\delta_{2} \leq 2$ then

$$
\delta_{2}=[2, \ldots],
$$

and inspecting Figure 1 we see that $X_{M}$ must contain a configuration of spheres dual to the graph of Figure 3. By blowing down (-1)-spheres, this immediately implies that $X_{M}$ contains an embedded sphere of square zero, which is impossible by Corollary 2.3

Assuming $\delta_{2}>2$, we have

$$
\delta_{2}=[m_{1}, \overbrace{2, \ldots, 2}^{m_{2}}, m_{3}, \ldots, m_{2 q-1}, \overbrace{2, \ldots, 2}^{m_{2 q}}],
$$




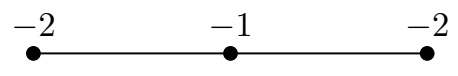

Figure 3: Configuration of spheres in $X_{M}$ when $\delta_{2} \leq 2$

for some

$$
m_{1}, m_{3}, \ldots, m_{2 q-1} \geq 3, \quad m_{2}, m_{4}, \ldots, m_{2 q} \geq 0 .
$$

Since $\delta_{3} \geq \delta_{2}$, we also have

$$
\delta_{3}=[d, \ldots], \quad \text { with } \quad d \geq 3 .
$$

\section{Blowing down and adjunction inequalities}

Since the central sphere in Figure 1 is a $(-1)$-sphere, we can blow it down. This shows that the 4 -manifold $X_{M}$ of Section 2 contains $X^{(0)} \# \overline{\mathbb{C P}}^{2}$, where $X^{(0)}$ is the plumbing associated to the graph of Figure 4 Blowing down $X^{(0)}$

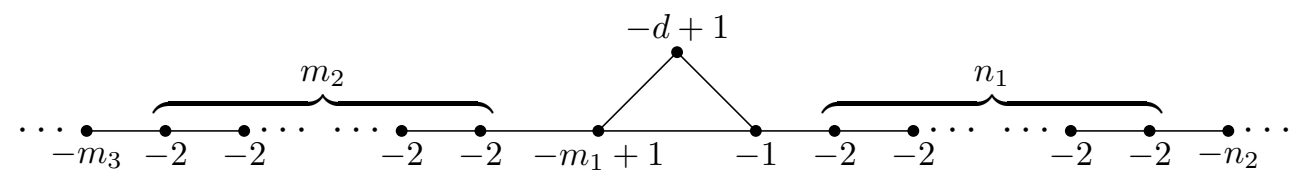

Figure 4: The manifold $X^{(0)}$

$n_{1}+1$ times, we obtain Figure 5] representing a four-manifold $X^{(1)}$ such that

$$
X^{(0)} \cong X^{(1)} \#\left(n_{1}+1\right) \overline{\mathbb{C P}}^{2} .
$$

Notice that each vertex in Figure 5 corresponds to an embedded sphere. In this picture and the following ones, a numerical weight of type " $(n)$ " on the edge between two vertices of the graph denotes the intersection number between suitably chosen homology classes corresponding to the vertices.

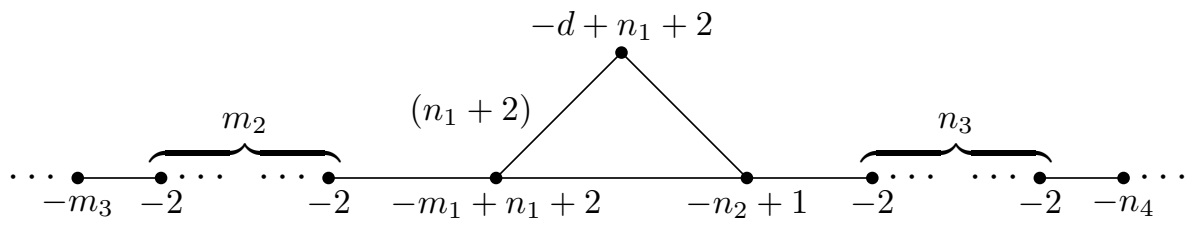

Figure 5: The manifold $X^{(1)}$ 
By Corollary 2.3. $X_{M}$ contains no spheres of non-negative self-intersection. Therefore, since $X^{(1)}$ can be embedded in $X_{M}$, we have

$$
-m_{1}+n_{1}+2 \leq-1 \text {. }
$$

If $-m_{1}+n_{1}+2=-1$ we blow down another $m_{2}+1$ times obtaining a $4-$ manifold $X^{(2)}$ such that

$$
X^{(1)} \cong X^{(2)} \#\left(m_{2}+1\right) \overline{\mathbb{C P}}^{2},
$$

with $X^{(2)}$ given by Figure 6] In Figure 6] the vertices on the horizontal line correspond to embedded spheres and the top vertex can be represented by an immersed sphere with $\left(m_{2}+1\right)\left(\begin{array}{c}n_{1}+2 \\ 2\end{array}\right)$ positive, transverse self-intersections. Observe that, by smoothing out the self-intersections, we can represent the same homology class by an embedded surface of genus

$$
g_{2}=\left(m_{2}+1\right)\left(\begin{array}{c}
n_{1}+2 \\
2
\end{array}\right) .
$$

Moreover, by Corollary [2.3 we have

$$
-n_{2}+m_{2}+2 \leq-1 .
$$

Continuing in the same fashion, this process gives rise to a sequence of mani-

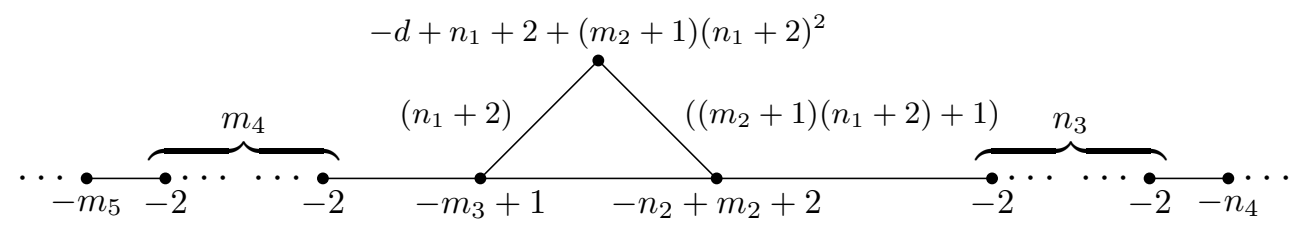

Figure 6: The manifold $X^{(2)}$

folds

$$
X^{(0)} \rightarrow X^{(1)} \rightarrow X^{(2)} \rightarrow \cdots \rightarrow X^{(i)} \rightarrow \cdots
$$

such that each $X^{(i)}$ (if defined) is given by Figure 7 for even $i$ and by Figure 8 for odd $i$. By Corollary 2.3, we have

$$
(-1)^{i}\left(m_{i}-n_{i}\right)+2 \leq-1
$$

for each $i$ such that $X^{(i)}$ is defined. Moreover, if $X^{(i)}$ is defined, then in order for $X^{(i+1)}$ to be defined as well we need to have

$$
(-1)^{i}\left(m_{i}-n_{i}\right)+2=-1 .
$$

If Equation (4.5) holds, then

$$
X^{(i)} \cong \begin{cases}X^{(i+1)} \#\left(n_{i+1}+1\right) \overline{\mathbb{C P}}^{2} & \text { if } i \text { is even } \\ X^{(i+1)} \#\left(m_{i+1}+1\right) \overline{\mathbb{C P}}^{2} & \text { if } i \text { is odd }\end{cases}
$$




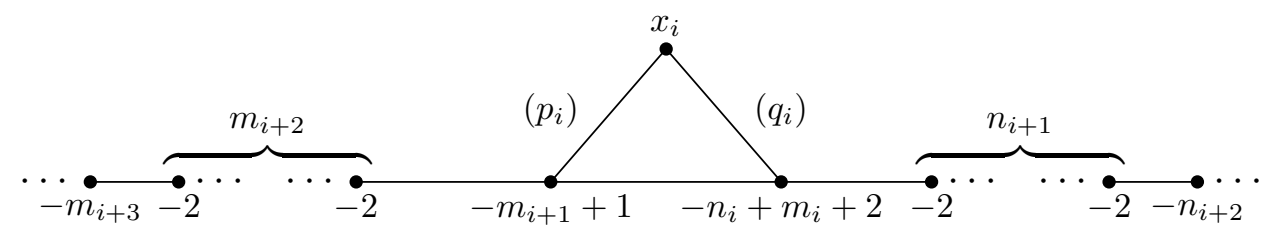

Figure 7: The manifold $X^{(i)}$ for even $i$

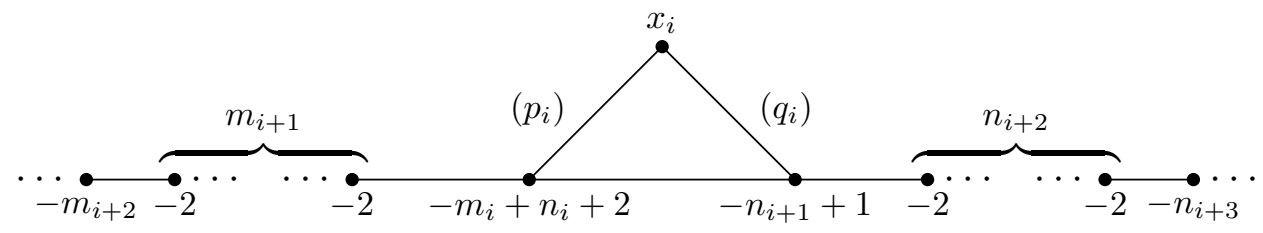

Figure 8: The manifold $X^{(i)}$ for odd $i$

As in the case of $X^{(2)}$, the homology class corresponding to the top vertex in each of Figures 7 and 8 can be represented in a natural way by an immersed sphere $S_{i} \subset X^{(i)}$. We denote by $g_{i}$ the genus of the smooth surface $\Sigma_{i}$ obtained by smoothing out the singularities of $S_{i}$. Then, the numbers $p_{i}$ and $q_{i}$ denote algebraic as well as geometric intersection numbers between $\Sigma_{i}$ and two embedded spheres representing the homology classes which correspond to the vertices connected to the top. Moreover, it is easy to check that the following relations hold:

$$
\begin{aligned}
& x_{i+1}= \begin{cases}x_{i}+\left(n_{i+1}+1\right) q_{i}^{2} & \text { if } i \text { is even } \\
x_{i}+\left(m_{i+1}+1\right) p_{i}^{2} & \text { if } i \text { is odd }\end{cases} \\
& p_{i+1}=\left\{\begin{array}{l}
p_{i}+\left(n_{i+1}+1\right) q_{i} \\
p_{i} \text { if } i \text { is even } i \text { is odd }
\end{array}\right. \\
& q_{i+1}= \begin{cases}q_{i} \text { if } i \text { is even } \\
q_{i}+\left(m_{i+1}+1\right) p_{i} & \text { if } i \text { is odd }\end{cases} \\
& g_{i+1}= \begin{cases}g_{i}+\left(n_{i+1}+1\right)\left(\begin{array}{c}
q_{i} \\
2
\end{array}\right) & \text { if } i \text { is even } \\
g_{i}+\left(m_{i+1}+1\right)\left(\begin{array}{c}
p_{i} \\
2
\end{array}\right) & \text { if } i \text { is odd }\end{cases}
\end{aligned}
$$

Strictly speaking, Figures 7 and 8 should be taken literally only if

$$
0 \leq i<\min (2 q, 2 p+1)
$$

(Recall that $p$ and $q$ were defined in Equations (4.3) and (4.4), respectively). When $i=\min (2 q, 2 p+1)$, the pictures should be suitably interpreted, because 
the diagram has only one horizontal leg. Similarly, Equations (4.6) shold be thought of as relations only when (4.7) holds, while for $i=\min (2 q, 2 p+1)$ they should be thought of as the definition of $x_{i+1}, p_{i+1}, q_{i+1}$ and $g_{i+1}$.

Using (4.6) it is easy to check that the number

$$
2 g_{i}-2-x_{i}+p_{i}+q_{i}, \quad 0 \leq i \leq \min (2 q+1,2 p+2),
$$

is independent of $i$, and therefore it is always equal to its value for $i=0$, i.e. $d-1$. On the other hand, the adjunction inequality implies

$$
2 g_{i}-2-x_{i} \geq 0
$$

hence

$$
d>p_{i}+q_{i}, \quad 0 \leq i \leq \min (2 q+1,2 p+2) .
$$

\section{The end of the proof}

By considering the Riemenschneider's point diagram $D\left(\delta_{2}, \delta_{2}^{\prime}\right)$, we see that

$$
\delta_{2}^{\prime}=[\overbrace{2, \ldots, 2}^{m_{1}-2}, m_{2}+3, \overbrace{2, \ldots, 2}^{m_{3}-3}, m_{4}+3, \ldots, \overbrace{2, \ldots, 2}^{m_{2 q-1}-3}, m_{2 q}+2] .
$$

Clearly, one of the following holds:

(1) There exists $k$ such that $0 \leq k \leq \min (2 q, 2 p+1)$, Equation (4.5) holds for every $0 \leq i<k$, and

$$
(-1)^{k}\left(m_{k}-n_{k}\right)+2<-1 .
$$

(2) Equation (4.5) holds for every $0 \leq i \leq \min (2 q, 2 p+1)$.

We shall now treat separately the possible cases which can occur.

\section{(1) holds and $k$ is even}

In this case we have

$$
\begin{gathered}
\delta_{2}^{\prime}<\overbrace{2, \ldots, 2}^{m_{1}-2}, m_{2}+3, \overbrace{2, \ldots, 2}^{m_{3}-3}, \ldots, m_{k-2}+3, \overbrace{2, \ldots, 2}^{m_{k-1}-3}, m_{k}+3] \leq \\
\leq[\overbrace{2, \ldots, 2}^{n_{1}+1}, n_{2}, \overbrace{2, \ldots, 2}^{n_{3}}, \ldots, n_{k-2}, \overbrace{2, \ldots, 2}^{n_{k-1}}, n_{i}-1]< \\
{[\overbrace{2, \ldots, 2}^{n_{1}+1}, n_{2}, \overbrace{2, \ldots, 2}^{n_{3}}, \ldots, n_{k-2}, \overbrace{2, \ldots, 2}^{n_{k-1}}, n_{i}, \ldots]=\delta_{1}}
\end{gathered}
$$


Lemma 4.3 Let $0 \leq k \leq \min (2 q+1,2 p+2)$, and suppose that Equation (4.5) holds for every $0 \leq i<k$. Then, if $k$ is even we have

$$
\frac{p_{k}+q_{k}}{p_{k}}=[m_{k}+3, \overbrace{2, \ldots, 2}^{m_{k-1}-3}, m_{k-2}+3, \ldots, \overbrace{2, \ldots, 2}^{m_{3}-3}, m_{2}+3, \overbrace{2, \ldots, 2}^{m_{1}-2}],
$$

while if $k$ is odd then

$$
\frac{p_{k}+q_{k}}{q_{k}}=[\overbrace{2, \ldots, 2}^{n_{k}+1}, n_{k-1}, \overbrace{2, \ldots, 2}^{n_{k-2}}, n_{k-3}, \ldots, \overbrace{2, \ldots, 2}^{n_{3}}, n_{2}, \overbrace{2, \ldots, 2}^{n_{1}+1}] .
$$

Proof Observe that Relations (4.6) imply

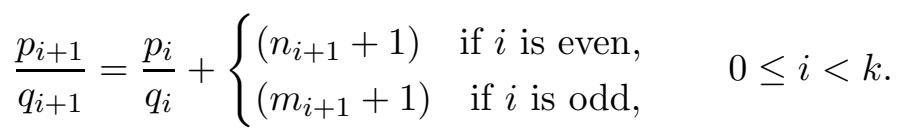

Let us introduce the notation:

$$
\left[n_{1}, \ldots, n_{k}\right]^{+}:=n_{1}+\frac{1}{n_{2}+\frac{1}{\ddots+\frac{1}{n_{k}}}}
$$

Since Equation (4.5) holds for every $0 \leq i<k$, by (4.9) we get, when $k$ is even,

$$
1+\frac{q_{k}}{p_{k}}=\left[m_{k}+2, m_{k-1}-2, m_{k-2}+1, \ldots, m_{3}-2, m_{2}+1, m_{1}-1\right]^{+},
$$

and when $i$ is odd

$$
1+\frac{p_{k}}{q_{k}}=\left[n_{k}+2, n_{k-1}-2, n_{k-2}+1, \ldots, n_{3}+1, n_{2}-1, n_{1}+2\right]^{+} .
$$

The lemma follows from the following identities, which can be established by a straightforward induction:

$$
\begin{gathered}
{\left[m_{k}+2, m_{k-1}-2, m_{k-2}+1, \ldots, m_{3}-2, m_{2}+1, m_{1}-1\right]^{+}=} \\
{[m_{k}+3, \overbrace{2, \ldots, 2}^{m_{k-1}-3}, m_{k-2}+3, \ldots, \overbrace{2, \ldots, 2}^{m_{3}-3}, m_{2}+3, \overbrace{2, \ldots, 2}^{m_{1}-2} .} \\
{\left[n_{k+1}+2, n_{k}-2, n_{k-1}+1, \ldots, n_{3}+1, n_{2}-1, n_{1}+2\right]^{+}=} \\
{[\overbrace{2, \ldots, 2}^{n_{k+1}+1}, n_{k}, \overbrace{2, \ldots, 2}^{n_{k-1}}, n_{k-2}, \ldots, \overbrace{2, \ldots, 2}^{n_{3}}, n_{2}, \overbrace{2, \ldots, 2}^{n_{1}+1} .}
\end{gathered}
$$


By (4.8) and Lemma 4.3. $\delta_{3}$ is bigger than the numerator of a fraction representing the number

$$
\rho=[\overbrace{2, \ldots, 2}^{m_{1}-2}, m_{2}+3, \overbrace{2, \ldots, 2}^{m_{3}-3}, \ldots, m_{k-2}+3, \overbrace{2, \ldots, 2}^{m_{k-1}-3}, m_{k}+3] .
$$

If $\rho=\frac{m}{a}$ with $a$ and $m$ coprime, then

$$
\delta_{1}>\frac{m}{a}, \quad \delta_{2}>\rho^{\prime}=\frac{m}{m-a} \quad \text { and } \quad \delta_{3}>m .
$$

Therefore $\Gamma(M)$ is realizable.

\section{(1) holds and $k$ is odd}

In this case we have

$$
\begin{aligned}
& \delta_{2}^{\prime}<\overbrace{2, \ldots, 2}^{m_{1}-2}, m_{2}+3, \overbrace{2, \ldots, 2}^{m_{3}-3}, \ldots, m_{k-3}+3, \overbrace{2, \ldots, 2}^{m_{k-2}-3}, m_{k-1}+3, \overbrace{2, \ldots, 2}^{m_{k}-3}] \leq \\
& \leq[\overbrace{2, \ldots, 2}^{n_{1}+1}, n_{2}, \overbrace{2, \ldots, 2}^{n_{3}}, \ldots, n_{k-3}, \overbrace{2, \ldots, 2}^{n_{k-2}}, n_{k-1}, \overbrace{2, \ldots, 2}^{n_{k}+1}]< \\
& \overbrace{2, \ldots, 2}^{n_{1}+1}, n_{2}, \overbrace{2, \ldots, 2}^{n_{3}}, \ldots, n_{k-3}, \overbrace{2, \ldots, 2}^{n_{k-2}}, n_{k-1}, \overbrace{2, \ldots, 2}^{n_{k}}, \ldots]=\delta_{1}
\end{aligned}
$$

By (4.8) and Lemma 4.3, $\delta_{3}$ is bigger than the numerator of

$$
\frac{m}{a}=[\overbrace{2, \ldots, 2}^{n_{1}+1}, n_{2}, \overbrace{2, \ldots, 2}^{n_{3}}, \ldots, n_{k-3}, \overbrace{2, \ldots, 2}^{n_{k}-2}, n_{k-1}, \overbrace{2, \ldots, 2}^{n_{k}+1}] .
$$

As in the previous case, it follows that $\Gamma(M)$ is realizable.

(2) holds and $2 q<2 p+1$

We have

$$
\begin{aligned}
\delta_{2}^{\prime}<\overbrace{2, \ldots, 2}^{m_{1}-2}, m_{2}+3, \ldots, m_{2 q}+3, \overbrace{2, \ldots, 2}^{n_{2 q+1}+1} & = \\
& =[\overbrace{2, \ldots, 2}^{n_{1}+1}, n_{2}, \ldots, n_{2 q}, \overbrace{2, \ldots, 2}^{n_{2 q+1+1}}]< \\
& <\overbrace{2, \ldots, 2}^{n_{1}+1}, n_{2}, \ldots, n_{2 q}, \overbrace{2, \ldots, 2}^{n_{2 q+1}}, \ldots]=\delta_{1} .
\end{aligned}
$$

By (4.8) and Lemma 4.3, $\delta_{3}$ is bigger than the numerator of

$$
\overbrace{2, \ldots, 2}^{n_{1}+1}, n_{2}, \ldots, n_{2 q}, \overbrace{2, \ldots, 2}^{n_{2 q+1}+1}]
$$

therefore $\Gamma(M)$ is realizable. 
(2) holds and $2 p+1<2 q$

We have

$$
\begin{aligned}
\delta_{2}^{\prime}<\overbrace{2, \ldots, 2}^{m_{1}-2}, & m_{2}+3, \ldots, m_{2 p}+3, \overbrace{2, \ldots, 2}^{m_{2 p+1}-3}, m_{2 p+2}+3]= \\
= & \overbrace{2, \ldots, 2}^{n_{1}+1}, n_{2}, \ldots, n_{2 p}, \overbrace{2, \ldots, 2}^{n_{2 p+1}}, m_{2 p+2}+3]< \\
& <\overbrace{2, \ldots, 2}^{n_{1}+1}, n_{2}, \ldots, n_{2 p}, \overbrace{2, \ldots, 2}^{n_{2 p+1}}]=\delta_{1} .
\end{aligned}
$$

By (4.8) and Lemma 4.3, $\delta_{3}$ is bigger than the numerator of

$$
\overbrace{2, \ldots, 2}^{m_{1}-2}, m_{2}+3, \ldots, m_{2 p}+3, \overbrace{2, \ldots, 2}^{m_{2 p+1}-3}, m_{2 p+2}+3],
$$

therefore $\Gamma(M)$ is realizable.

\section{References}

[1] D. Eisenbud, U. Hirsch and W.D. Neumann, Transverse foliations of Seifert bundles and self homeomophism of the circle, Comment. Math. Helvetici 56 (1981) 638-660. MathReview

[2] Y Eliashberg, Filling by holomorphic discs and its applications, Geometry of low-dimensional manifolds, 2 (Durham, 1989), London Math. Soc. Lecture Notes Series 151, Cambridge Univ. Press (1990) 45-67. MathReview

[3] Y. Eliashberg, A few remarks about symplectic filling, Geom. Topol. 8 (2004) 277-293. MathReview

[4] Y. Eliashberg and W. Thurston, Confoliations, University Lecture Series, 13, American Mathematical Society, Providence, RI, 1998. MathReview

[5] J.B. Etnyre and K. Honda, Tight contact structures with no symplectic fillings, Invent. Math. 148 (2002) no. 3, 609-626. MathReview

[6] E. Giroux, Structures de contact sur les variétés fibrées en cercles audessus d'une surface, Comment. Math. Helv. 76 (2001) no. 2, 218-262. MathReview

[7] K. Honda Confoliations transverse to vector fields, preprint, preliminary version: http://math.usc.edu/ khonda/research.html

[8] J. Hempel 3-Manifolds, Ann. of Math. Studies 86, Princeton Univ. Press, Princeton, N.J., 1976. MathReview

[9] M. Jankins and W.D. Neumann, Homomorphisms of Fuchsian groups to PSL $(2, R)$, Comment. Math. Helv. 60 (1985) no. 3, 480-495. MathReview 
[10] M. Jankins and W.D. Neumann, Rotation Numbers of Products of Circle Homeomorphisms, Math. Ann. 271 (1985) 381-400. MathReview

[11] P.B. Kronheimer, T.S. Mrowka, Monopoles and contact structures, Invent. Math. 130 (1997) 209-256. MathReview

[12] J. Milnor On the existence of a connection with curvature zero, Comment. Math. Helv. 32 (1958) 215-223. MathReview

[13] J.D. McCarthy, J.G. Wolfson, Symplectic gluing along hypersurfaces and resolution of isolated orbifold singularities, Invent. Math. 119 (1995) no. 1, 129-154. MathReview

[14] R. Naimi, Foliations transverse to fibers of Seifert manifolds, Comment. Math. Helv. 69 (1994) no. 1, 155-162. MathReview

[15] W.D. Neumann and F. Raymond, Seifert manifolds, plumbing, $\mu$ invariant and orientation reversing maps, Algebraic and geometric topology (Santa Barbara 1977), Lecture Notes in Math. 664, Springer (1978) 163-196. MathReview

[16] P. Orlik, Seifert manifolds, Lecture Notes in Mathematics, Vol. 291. Springer 1972. MathReview

[17] P. Ozsváth, Z. Szabó, The symplectic Thom conjecture, Ann. of Math. (2) 151 (2000) no. 1, 93-124. MathReview

[18] O. Riemenschneider, Deformationen von Quotientensingularitäten (nach zyklischen Gruppen), Math. Ann. 209 (1974) 211-248. MathReview

[19] A. Sato, T. Tsuboi, Contact structures on closed manifolds fibered by the circles, Mem. Inst. Sci. Tech. Meiji Univ. 33 (1994) 41-46.

[20] J. Wood, Bundles with totally disconnected structure group, Comment. Math. Helv. 40 (1971) 257-279. MathReview

Dipartimento di Matematica "L. Tonelli"

Università di Pisa

I-56127 Pisa, ITALY

and

Department of Mathematics

University of Georgia

Athens, GA 30602, USA

Email: lisca@dm.unipi.it, gordana@math.uga.edu

Received: 21 June 2004 